\title{
Do Not Tunnel the Small Bowel during the Tunneling of a Femoro-Femoral Bypass
}

\author{
Chris Tae Young Chung, Sangil Min, and Seung-Kee Min \\ Division of Vascular Surgery, Department of Surgery, Seoul National University College of Medicine, Seoul, Korea
}

\begin{abstract}
A 62-year-old male with a history of femoro-femoral crossover bypass surgery 31 months previously presented with recurrent symptoms of fever and chills, along with a previous positive blood culture. Computed tomography showed vegetation in the bypass graft located in the peritoneal cavity, closely abutting the small bowel and soft tissue lesions in the right proximal thigh and distal calf. Under high suspicion of graft-enteric fistula with metastatic infection, surgery was performed to remove the previous graft and insert a new femoro-femoral bypass graft subcutaneously. Small bowel resection and anastomosis were also performed because the graft penetrated the small bowel mesentery and eroded into the small bowel. The patient had a patent graft without infection for more than 10 years. This case demonstrates the importance of tunneling in femoro-femoral crossover bypass free from the small bowel or other intraperitoneal organs.
\end{abstract}

Key Words: Vascular grafting, Postoperative complications, Prosthesis-related infections
Received May 13, 2021

Revised June 10, 2021

Accepted June 16, 2021

Published on June 28, 2021

Corresponding author: Seung-Kee Min Division of Vascular Surgery, Rm 5313, Biomedical Research Institute, Seoul National University Hospital, 101 Daehakro, Jongno-gu, Seoul 03080, Korea

Tel: 82-2-2072-0297

Fax: 82-2-766-3975

E-mail: skminmd@snuh.org https://orcid.org/0000-0002-1433-2562

Copyright ( $\odot 2021$ The Korean Society for Vascular Surgery

This is an Open Access article distributed under the terms of the Creative Commons Attribution Non-Commercial license (http://creativecommons.org/licenses/by-nc/4.0) which permits unrestricted non-commercial use, distribution, and reproduction in any medium, provided the original work is properly cited.

Vasc Specialist Int • https://doi.org/10.5758/vsi.210036

\section{INTRODUCTION}

Perforation of the small bowel during a femoro-femoral crossover bypass graft is a very rare complication. However, it can cause severe morbidity or mortality if not treated properly. For educational purposes, we herein report a rare case of small bowel perforation after inaccurate tunneling during the crossover bypass, presenting with metastatic infection. This study was approved by the Institutional Review Board (IRB No: C-2102-107-1197) and the requirement for patient consent was waived.

\section{CASE}

A 62-year-old male presented to the emergency room because of a 10-day history of recurrent fever and chills. Two months prior, he had a fever and positive blood culture for Escherichia coli and was treated with antibiotics.
His body temperature was $38.8^{\circ} \mathrm{C}$, and blood tests revealed leukocytosis $\left(22,570 / \mathrm{mm}^{3}\right)$. He underwent right femoroleft femoral crossover bypass surgery at another hospital 31 months prior. Computed tomography revealed soft tissue lesions in the right proximal thigh, distal calf, and vegetation in the bypass graft, which seemed to be located below the peritoneum (Fig. 1). Positron emission tomography revealed multiple hypermetabolic lesions in the bypass graft, right upper thigh, and lower calf (Fig. 2). Ultrasoundguided aspiration of the thigh mass revealed a dark red pus, which was positive for E. coli. A graft-enteric fistula with metastatic infection was suspected. After administration of systemic antibiotics, incision and drainage of the pus was performed by an orthopedic surgeon, and the culture showed mixed infection with E. coli and Streptococcus species. Seven days after antibiotic therapy ( 5 days after the drainage), negative conversion of the blood culture was confirmed, and vascular surgery was performed. Ini- 


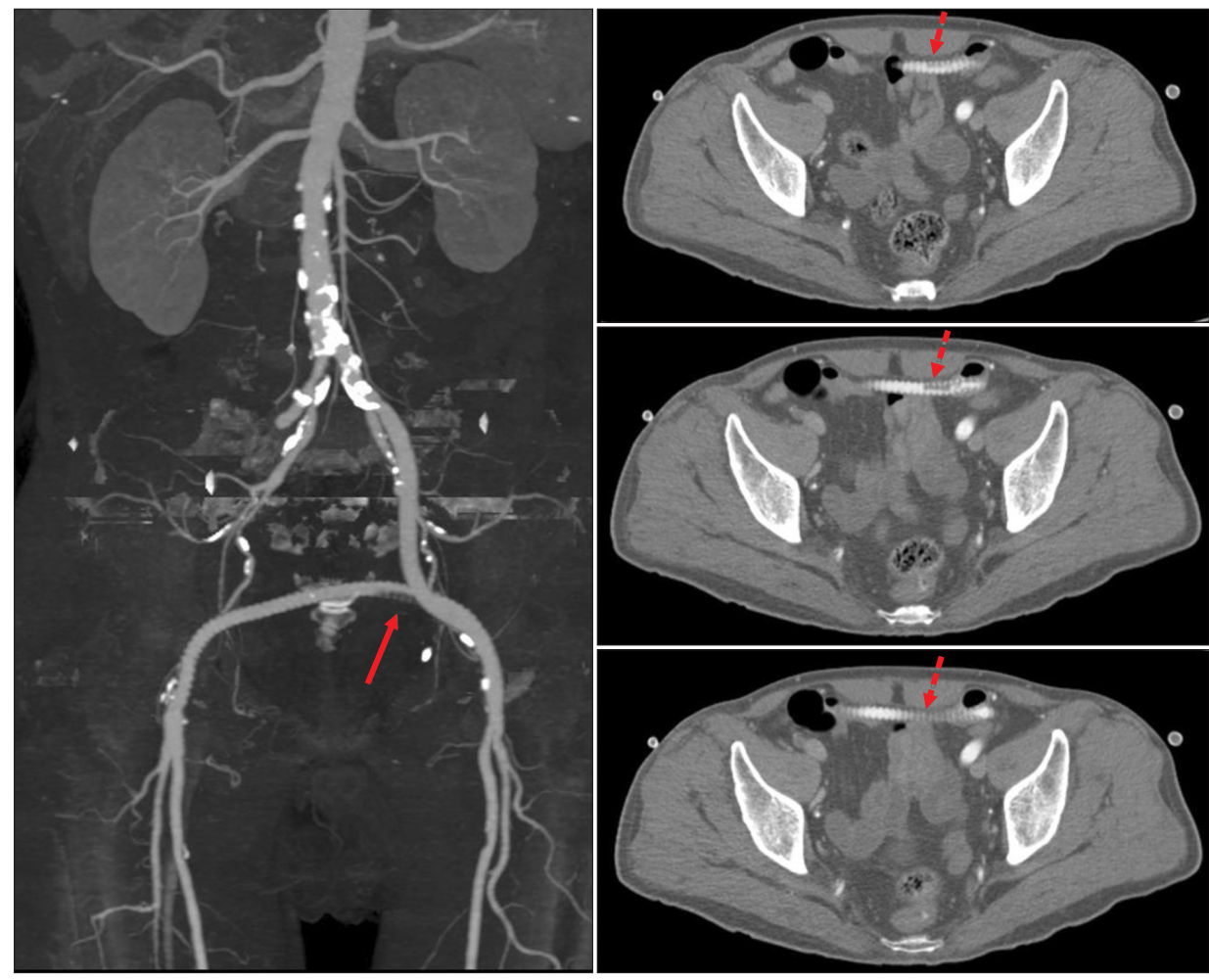

Fig. 1. Computed tomography angiography showed vegetation in the bypass graft (red arrow), and axial views showed the bypass graft inside the peritoneum (red dotted arrow) with close contact with the small bowel.

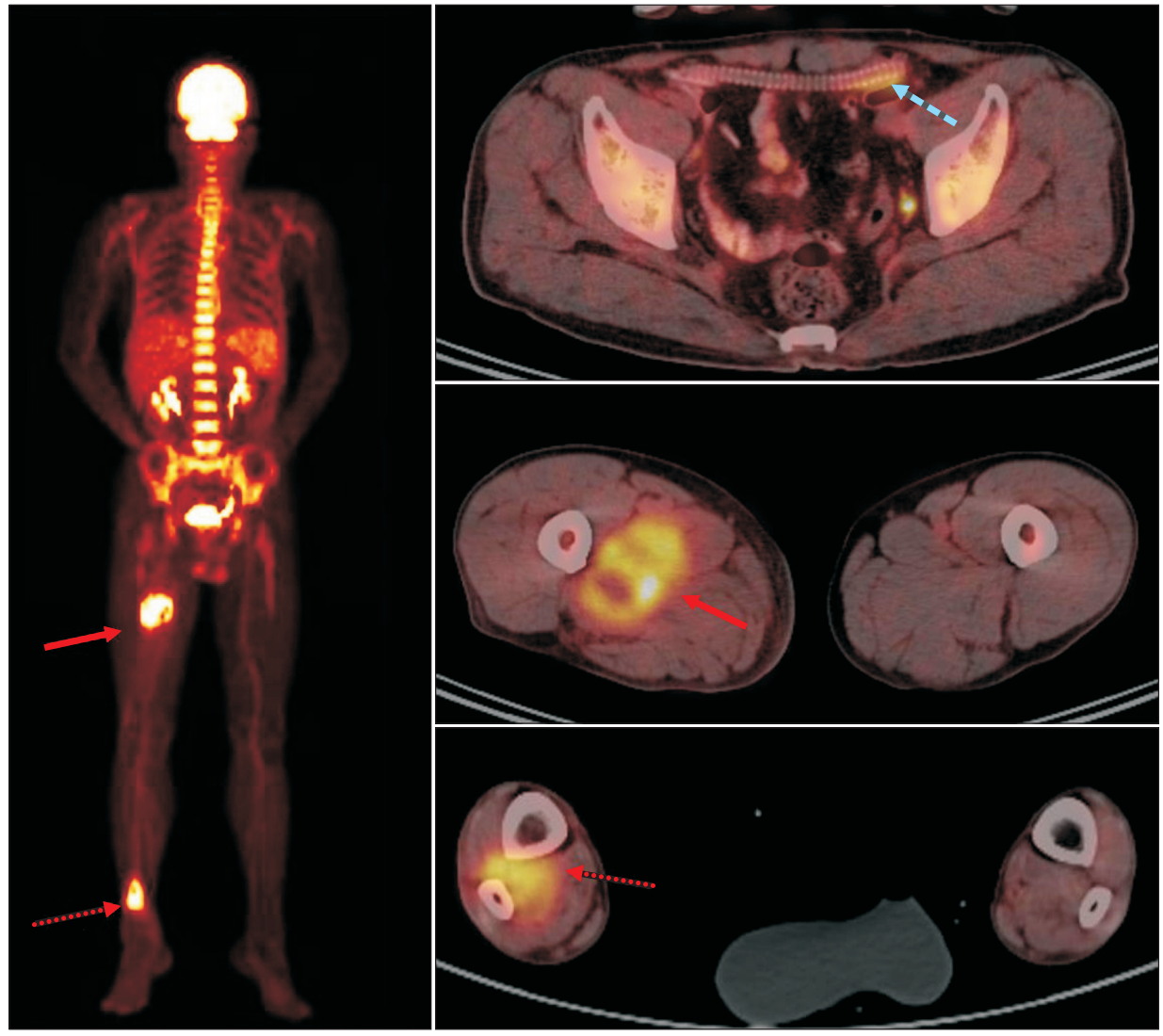

Fig. 2. Positron emission tomography showed multiple hypermetabolic lesions in the bypass graft (blue dashed arrow), right upper thigh (red arrow), and lower calf (red dotted arrow). 


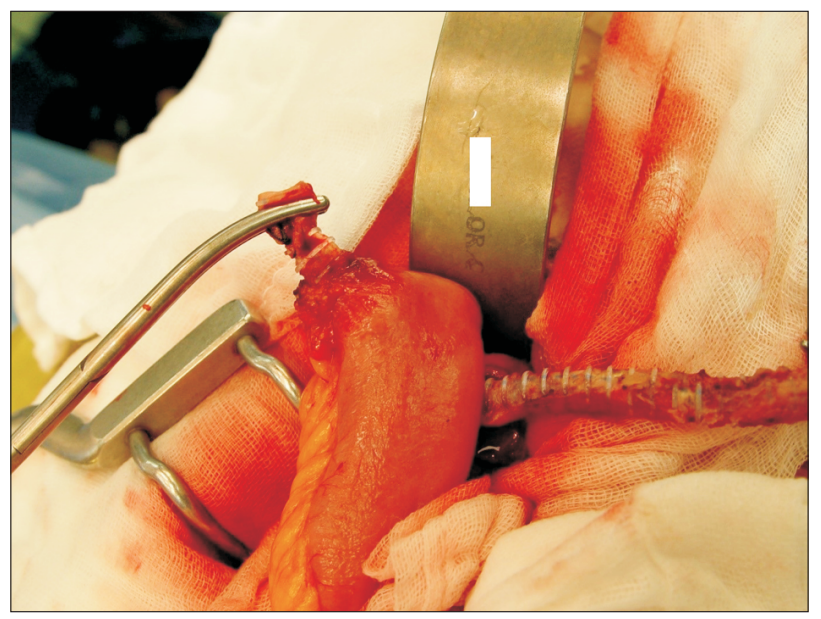

Fig. 3. Operative picture showed the prosthetic graft penetrating the small bowel mesentery and eroding the small bowel.

tially, both inguinal areas were dissected, and no signs of infection were observed. Therefore, a new femoro-femoral bypass graft was inserted via a new subcutaneous tunnel with a ringed expanded polytetrafluoroethylene (ePTFE) graft. The previous grafts in the inguinal area were resected and sent for culture, which resulted in no growth of microorganisms. An additional lower midline incision was made, revealing the previous graft perforating the small bowel mesentery and eroding into the small bowel, $160 \mathrm{~cm}$ proximal from the ileocecal valve, compatible with graftenteric erosion (Fig. 3). The infected graft was removed and a 5-cm section of the small bowel was resected and anastomosed in an end-to-end fashion. Bilateral defects in the peritoneum in both suprainguinal areas were repaired. Postoperative antibiotics were administered for four weeks. The patient recovered well with no postoperative events and remained in good health with a patent graft without infection for more than 10 years.

\section{DISCUSSION}

Only three cases of bowel perforation during femorofemoral crossover bypass have been reported in the literature. Hinchliffe et al. [1] reported a case series of 231 patients who underwent femoro-femoral bypass grafting after aorto-uni-iliac endovascular aneurysm repair. They reported two cases of graft sepsis due to visceral damage that developed during the tunneling of a prosthetic graft. One patient had an unrecognized incisional hernia from a previous laparotomy, and the other patient had previously undergone laparotomy to treat an intra-abdominal abscess. In patients with previous laparotomy, especially in the su- prapubic area near the expected tunneling course, preoperative evaluation of incisional hernia or concealed abscess is necessary.

van Nieuwenhuizen et al. [2] reported a case of fatal sepsis due to small bowel perforation that occurred two days after femoro-femoral bypass grafting. They intended to create a subcutaneous tunnel, but the tunneling was performed half-blind using a stump clamp, inadvertently perforating the small bowel; the patient succumbed to a poorly understood sepsis, the cause of which was discovered by autopsy. They warned that blind tunneling could perforate the peritoneum and small bowel, especially in patients with extremely slim habitus. This is a case of definite technical failure during the tunneling procedure. We are grateful to van Nieuwenhuizen et al. for their candid report, revealing their mistakes in order to raise awareness in other vascular surgeons. We report this unusual case for the same educational purpose.

Another complication caused by femoro-femoral bypass tunneling was reported by Häcker et al. [3], who reported a graft tunneled through the urinary bladder. The graft inadvertently placed in the bladder caused self-limiting macrohematuria and later dysuria. Cystography revealed a misplaced graft 3 months after surgery. Interestingly, the graft was not infected and showed good patency and was extravesically relocated.

The tunneling technique of the femoro-femoral bypass graft is well described in a classical surgical atlas [4]. In brief, a suprapubic subcutaneous tunnel is digitally developed through both inguinal incisions. The aorta clamp is inserted via the left leg tunnel with the surgeon's right hand, and the clamp tip meets the surgeon's left fingertip, which guides the clamp out through the right inguinal wound. The midline fascial attachments are pierced with the aortic clamp, and the graft is pulled through the tunnel without twists or kinks in an inverted C configuration. The graft should be located in the deep subcutaneous layer and not below the inguinal ligament. Blind tunneling with any sharp instrument easily leads to perforation of the peritoneum, bladder, or intestine, especially in elderly patients. Careful guidance using fingertips during tunneling is easy and safe.

One more technically important issue is that any prosthetic graft should not make direct contact with the bowel. If the bowel contacts a graft, graft-enteric erosion eventually occurs. Honda et al. [5] reported that a ringed ePTFE graft in the peritoneum after a femoro-superior mesenteric artery bypass migrated into the small bowel. The prosthetic graft should be placed in the retroperitoneum. If part of the graft is located intraperitoneally and inevitably exposed to the small bowel, it should be covered with the omentum to 
prevent graft-enteric erosion or fistula.

Another example of bowel injury during tunneling can be seen in aortobifemoral bypass grafting. Blank et al. [6] reported aortic graft infections secondary to iatrogenic transcolonic graft malposition. During the tunneling of the aortobifemoral bypass, two graft limbs were malpositioned into the sigmoid colon and cecum in two cases. This could be avoided by tunneling under the guidance of the finger, which advances just above the native iliac artery. This technique can prevent the common tunneling failure of entrapping the ureter between the graft and iliac limb when the graft should be placed below the ureter and above the native artery.

To avoid recurrent graft infection, the operation sequence in this case was carefully designed. To avoid bloodborne reinfection, vascular surgery was performed after confirming negative conversion of blood cultures. After revealing that both inguinal anastomoses were intact and free of graft infection, a new femoro-femoral bypass was performed in situ, and as much of the previous graft was excised as possible. After closing both inguinal wounds, a lower midline incision was made, and small bowel resection and infected graft removal were performed in the last stage.

\section{CONFLICTS OF INTEREST}

The authors have nothing to disclose.

\section{ORCID}

\author{
Chris Tae Young Chung \\ http://orcid.org/0000-0003-4089-8276 \\ Sangil Min \\ http://orcid.org/0000-0002-0688-0278 \\ Seung-Kee Min \\ https://orcid.org/0000-0002-1433-2562
}

\section{AUTHOR CONTRIBUTIONS}

Concept and design: SKM. Analysis and interpretation: CTYC. Data collection: CTYC. Writing the article: CTYC, SKM. Critical revision of the article: SM. Final approval of the article: all authors. Statistical analysis: none. Obtained funding: none. Overall responsibility: SKM.

\section{REFERENCES}

1) Hinchliffe RJ, Alric P, Wenham PW, Hopkinson BR. Durability of femorofemoral bypass grafting after aortouniiliac endovascular aneurysm repair. J Vasc Surg 2003;38:498-503.

2) van Nieuwenhuizen RC, Kox C, Legemate DA. Small bowel perforation due to tunnelling of a femorofemoral crossover bypass. EJVES Extra 2007;13:21-22.
3) Häcker A, Uysal Z, Badawi K, Langbein $S$, Kamp S, Hatzinger $M$, et al. [Foreign body in the urinary bladder after vascular surgery]. Aktuelle Urol 2005;36:249-251. German.

4) Ouriel K, Rutherford RB. Atlas of vascular surgery: operative procedures. Philadelphia: W.B. Saunders Company; 1998. p. 84-85.

5) Honda K, Okamura Y, Nishimura Y,
Uchita S. Migration of the ringed ePTFE graft into the small intestine. J Vasc Surg 2013;57:525.

6) Blank JJ, Rothstein AE, Lee CJ, Malinowski MJ, Lewis BD, Ridolfi TJ, et al. Aortic graft infection secondary to iatrogenic transcolonic graft malposition. Vasc Endovascular Surg 2018;52:386-390. 